\section{Der Umgang mit Barrieren bei der Einführung von Pervasive Computing Ein Literaturüberblick}

von Andreas Graefe, Carsten Orwat, ITAS, und Timm Faulwasser, Universität Magdeburg

Pervasive Computing hält Einzug in nahezu alle Bereiche der medizinischen Versorgung. Dieser Artikel präsentiert ausgewählte Ergebnisse einer Analyse der internationalen Literatur, die im Rahmen des Forschungsprojektes PerCoMed ${ }^{1}$ durchgeführte wurde, und liefert einen Überblick zu typischen Anwendungsbereichen von Pervasive Computing im Gesundheitswesen. Ziel ist es, dem Leser einen komprimierten Einstieg in dieses komplexe Anwendungsfeld zu ermöglichen. Für die detaillierte Beschreibung des methodischen Vorgehens, die umfassende Analyse der Ergebnisse sowie sämtliche Referenzen verweisen wir auf Orwat et al. (2008).

\section{Literaturanalyse als Methode der Technikfolgenabschätzung}

Mehr noch als Wissenschaftler einer speziellen Disziplin stehen Technikfolgenabschätzer in regelmäßigen Abständen vor der Herausforderung, sich schnell und umfassend in neue Themengebiete einzuarbeiten. Insbesondere für neu aufstrebende Forschungs- und Technikgebiete sind wissenschaftliche Arbeiten aber oftmals über verschiedene Disziplinen verstreut oder werden kontrovers diskutiert. Dies macht es schwierig bis unmöglich, auf bereits bestehende Arbeiten zurückzugreifen, um einen konsolidierten Überblick über den aktuellen Stand der Forschung zu gewinnen.

Eine Methode, die dafür geeignet scheint, sich strukturiert in neue Themengebiete einzuarbeiten, ist die Literaturanalyse. Das Anfertigen einer Literaturanalyse setzt systematisches Vorgehen voraus und zwingt den Autor, sich intensiv mit dem Thema auseinanderzusetzen. Dies umfasst eine klare Definition und Abgrenzung zu verwandten Themengebieten, die Ableitung von Schlüsselbegriffen zur
Identifikation relevanter Artikel sowie die Ausarbeitung von Schemata zur Kategorisierung und Analyse von Ergebnissen. Insbesondere in der Anfangsphase von Projekten hilft das Instrument der Literaturanalyse, einen Überblick über Anwendungsmöglichkeiten von neuen Technologien sowie potenzielle Problembereiche zu gewinnen. Darüber hinaus liefern Literaturanalysen einen wichtigen Dienst für Wissenschaft und Praxis: Ein Überblick über den aktuellen Stand der Forschung schließt die Lücke zwischen einer unüberschaubaren Anzahl von Artikeln und dem Leser, der weder über die Zeit noch die Ressourcen verfügt, diese ad hoc zu überblicken.

\section{Methodisches Vorgehen}

Für den Zeitraum 2002 bis 2006 wurden Datenbankrecherchen sowie manuelle Recherchen in Inhaltsverzeichnissen und Kurzfassungen relevanter Fachzeitschriften nach englischsprachigen Artikeln durchgeführt, welche über Prototypen, Fallstudien oder klinische Studien aus dem Bereich des Pervasive Computing im Gesundheitswesen berichteten. Mit Hilfe eines zweistufigen Auswahl- und Analyseprozesses wurden letztendlich 69 relevante Artikel identifiziert, die wiederum 67 unterschiedliche Systeme beschreiben (siehe Abb. 1). Diese 67 Systeme bilden die Basis für die nachfolgenden Auswertungen. Davon wurden 24 in den USA implementiert, acht in Großbritannien, je vier in Frankreich und Taiwan sowie jeweils drei in Australien, Dänemark, Deutschland und Spanien. Insgesamt wurden 31 Systeme innerhalb der Europäischen Union implementiert. Zwei kommerziell vertriebene Systeme wurden zum Zeitpunkt der Analyse bereits weltweit eingesetzt. 
Abb. 1: Methodisches Vorgehen zur Identifikation der relevanten Artikel und Systeme

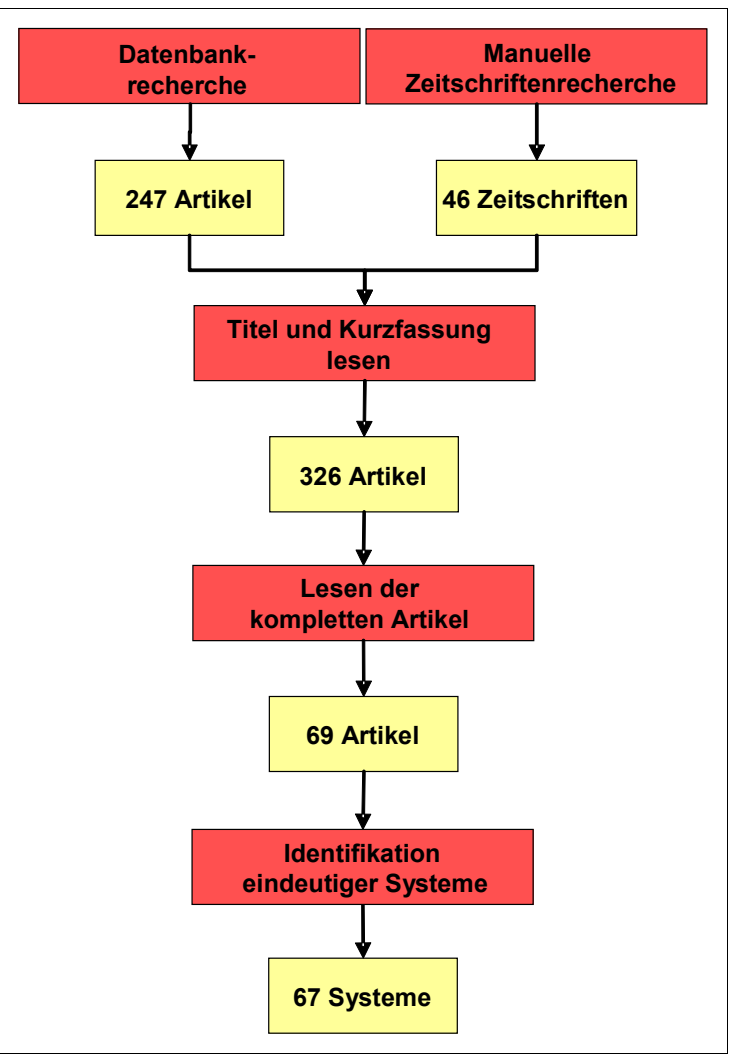

Quelle: Eigene Darstellung

\section{Quantitative Auswertung}

\subsection{Ergebnisüberblick}

Im Folgenden präsentieren wir ausgewählte Ergebnisse der quantitativen Analyse im Hinblick auf den Status der Systementwicklung, typische Einsatzfelder sowie Ziele der Systeme. ${ }^{3}$ Diese Auswertungen helfen, einen ersten Über- blick über den Stand der Forschung im Bereich Pervasive Computing zu gewinnen. Anschließend diskutieren wir einige - speziell für die Technikfolgenabschätzung interessante - Aspekte, die als kritische Punkte der Systemeinführung identifiziert werden konnten. Für den tieferen Einstieg ins Thema empfehlen wir die Lektüre von Fachzeitschriften, die wir in unserer Analyse als führend identifizieren konnten, $d$. h. die die höchste Anzahl von relevanten Artikeln veröffentlicht haben (vgl. Abb. 2).

\section{Status der Systementwicklung}

Unsere Analyse der empirischen Studien umfasst drei Stadien der Systementwicklung und -einführung: Prototypen bzw. Pilotstudien, klinische Studien sowie regulär eingesetzte Systeme. Abbildung 3 illustriert, dass sich der Großteil der Systeme (56) noch im Prototypstatus befindet, wohingegen nur sechs Systeme zum Zeitpunkt der Veröffentlichung ihrer Beschreibung bereits klinische Tests durchlaufen hatten. Fünf Systeme befanden sich bereits im regulären Einsatz. Wir schließen aus diesen Ergebnissen, dass Pervasive Computing noch am Anfang seiner Entwicklung steht und noch nicht von einem breiten Einsatz im Gesundheitswesen gesprochen werden kann. Als mögliche Gründe sehen wir Einführungshindernisse sozialer, organisatorischer und finanzieller Art. Die im Rahmen der Analyse gewonnenen Erkenntnisse im Hinblick auf hemmende Faktoren einer breiten Einführung von Pervasive Computing im Gesundheitswesen werden in Kapitel 5 diskutiert.

Abb. 2: Anzahl der ausgewählten Artikel (nach Zeitschriften)

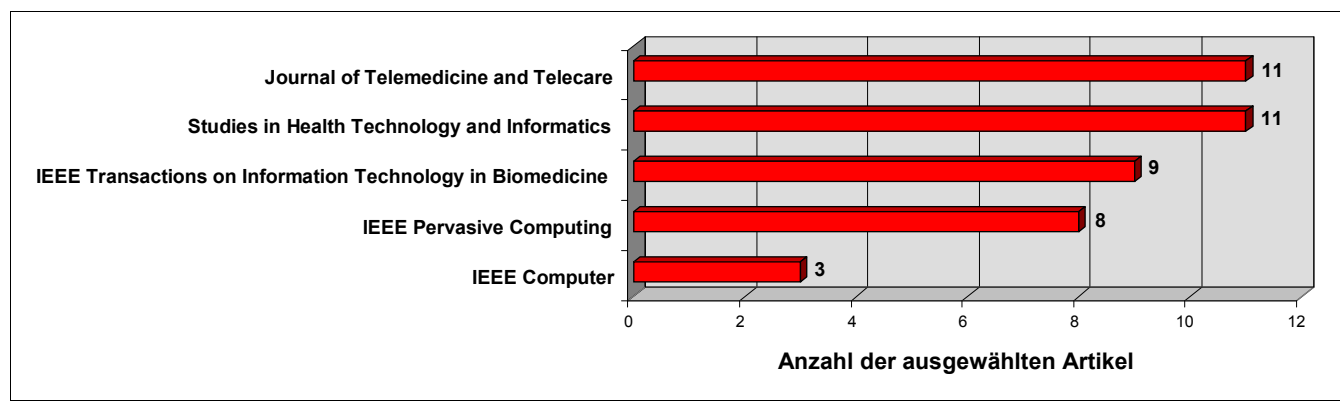

Quelle: Eigene Darstellung 
Abb. 3: Status der Systementwicklung

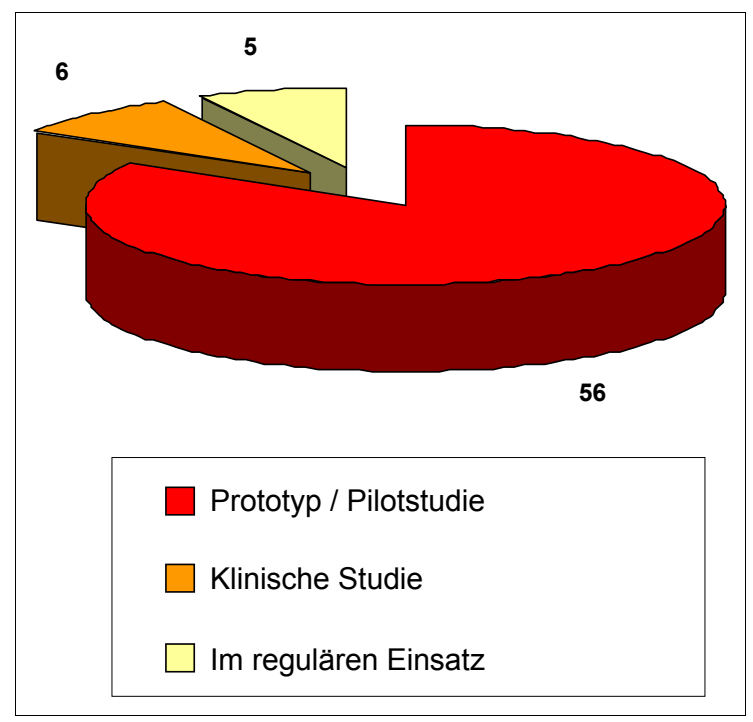

Quelle: Eigene Darstellung

\section{Einsatzfelder des Pervasive Computing}

Eine Auswertung nach den Einsatzfeldern der Systeme verdeutlicht, dass der Großteil der Systeme für den Einsatz in der häuslichen und mobilen (38) sowie in der klinischen Versorgung (24) bestimmt ist. Dieses Ergebnis deckt sich mit der verbreiteten Erwartung, dass Pervasive Computing durch die Möglichkeit der Fernüberwachung der Gesundheitsdaten von Patienten sowie durch Koordination von Arbeitsabläufen in komplexen Einrichtungen wie Kliniken hohe Verbesserungspotenziale zugerechnet wer- den. Vergleichsweise gering erscheint dagegen der Einsatz von Systemen in der Notfall- (7) und ambulanten Versorgung (4) sowie in Pflegeeinrichtungen (5). Für den Einsatz in Rehabilitationseinrichtungen konnte kein System identifiziert werden. Für diese wie für die folgende Auswertung ist zu beachten, dass sich die Anzahl der Systeme in den verschiedenen Kategorien nicht auf 67 addiert. Dies folgt aus der Tatsache, dass Systeme sowohl in unterschiedlichen Bereichen zum Einsatz kommen als auch mit ihnen mehrere Ziele - im Sinne von Einsatzzwecken - verfolgt werden können.

\section{Systemziele}

Die Entwicklung und der Einsatz neuer Technologien in der Gesundheitsversorgung sind üblicherweise mit der Absicht verbunden, organisatorische und/oder medizinische Verbesserungen herbeizuführen. Letztere lassen sich dabei weiter untergliedern in angestrebte Verbesserungen, die therapeutischen Zwecken dienen und solchen, die auf Prävention und Pflege ausgerichtet sind. Abbildung 5 zeigt, dass mit dem Großteil der Systeme (42) medizinische Verbesserungen im Bereich der Prävention und Pflege verfolgt werden, mit nur acht Systemen wird darauf abgezielt, die Therapie zu verbessern. Weiterhin sind 26 Systeme auf organisatorische Verbesserungen (wie beispielsweise Prozessautomatisierung und -kontrolle) oder auch auf unterstützende Aktivitäten (wie spezifische In-

\section{Abb. 4: Einsatzbereiche der Systeme}

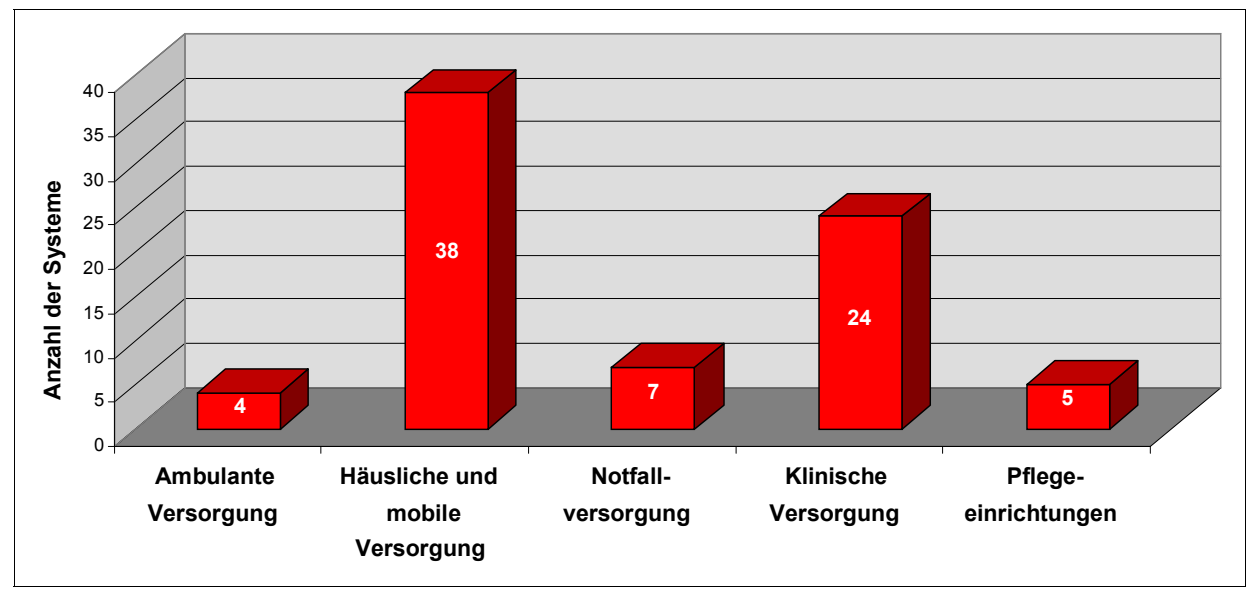

Quelle: Eigene Darstellung 


\section{Abb. 5: Ziele der Systeme}

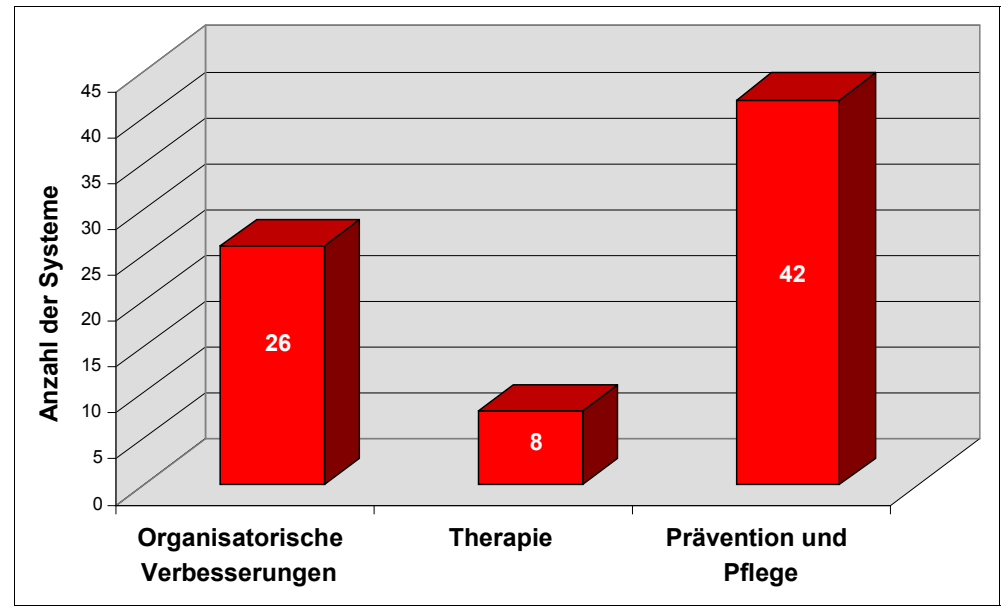

Quelle: Eigene Darstellung

formationsbereitstellung oder automatische Dokumentation von Arbeitsabläufen).

\subsection{Faktoren, die die Einführung beeinflussen}

Neben der quantitativen Auswertung und Systemkategorisierung, wurde eine qualitative Analyse mit dem Ziel der Identifikation kritischer Punkte und möglicher Hindernisse in der Einführung der Systeme durchgeführt. Hierbei wurde unterschieden zwischen organisatorischen, persönlichen und finanziellen Hindernissen sowie Barrieren aufgrund von Bedenken im Hinblick auf Verletzungen der Privatsphäre und des Datenschutzes. Überraschenderweise konnte nur eine geringe Anzahl von Artikeln identifiziert werden, die sich zumindest rudimentär mit möglichen Einführungshindernissen in der späteren Systemeinführung auseinander setzen. Ganze 19 Artikel schenken derartigen Problemen dagegen keinerlei Beachtung. Im Folgenden beschränkt sich die Darstellung auf die von Autoren berichteten Erfahrungen mit organisatorischen Rahmenbedingungen und Bedenken hinsichtlich Verletzungen von Privatsphäre und Datenschutz.

\section{Organisatorische Rahmenbedingungen}

Erkenntnisse über organisatorische oder persönliche Einführungsbarrieren konnten aus den Beschreibungen von sechs Systemen abgeleitet werden. Beispielsweise berichtet Dahl (2006) über positive Erfahrungen bei der Integration von digitalen Notizen in innerklinischen Arbeitsabläufen, die durch ortsspezifische Nachrichten auf PDAs („Personal Digital Assistants" bzw. „Handhelds") realisiert wurden. So führte der Einsatz dieses Systems zu einer verbesserten Kommunikation zwischen den Mitarbeitern der Klinik und reduzierte Unterbrechungen in Routineaktivitäten, da sich die Technologie aufgrund ihrer Flexibilität besser an den hohen Mobilitätsgrad und die ereignisgesteuerte Arbeitsumgebung in Kliniken anpassen konnte als traditionelle Kommunikationstechnologien wie Fax oder Telefon.

In einer Studie über ein System zur Überwachung von Vitalparametern von Patienten verdeutlichen Dadd et al. (2002) die Notwendigkeit spezifischer Designüberlegungen in Abhängigkeit von organisatorischen Rahmenbedingungen, unter denen die Technologie zum Einsatz kommt. Während die Überwachung von Patienten in deren häuslicher Umgebung langfristig angelegt ist und vergleichsweise wenig Akteure in den Prozess involviert, sind im klinischen Umfeld sowohl eine Vielzahl von Akteuren (wie Klinikpersonal oder Techniker), aber auch Geräte in den Versorgungsprozess eingebunden. Diese erhöhte Komplexität muss bei der Entwicklung von Systemen, welche sowohl im häuslichen als auch im klinischen Bereich Anwendung finden sollen, frühzeitig berücksichtigt werden 
In ihrer Analyse der Einführung eines Systems zur drahtlosen Messung des Blutdrucks während Operationen identifizieren Oyri et al. (2006) Einführungshindernisse aufgrund von Defiziten in der Ausbildung des Klinikpersonals. Insbesondere führe ein mangelndes Technologieverständnis zu einer ablehnenden Einstellung der Krankenschwestern gegenüber technologischen Neuerungen bzw. Veränderungen der Arbeitsabläufe. Als Schlussfolgerung empfehlen die Autoren Veränderungen in der Ausbildung des Klinikpersonals hin zu einem stärkeren Fokus auf Medizininformatik.

Reddy et al. (2003) untersuchen organisatorische Schwierigkeiten beim Einsatz von Pager-Systemen in der ambulanten Chirurgie, welche im Falle kritischer Laborergebnisse, möglicher Probleme bei der medikamentösen Behandlung oder negativer Entwicklungen der Gesundheitsparameter von Patienten automatisch Alarm auslösen. Letztendlich stieß das System durch den erzeugten Informationsüberfluss bei den Ärzten auf Ablehnung. Beispielsweise konnte das System nicht zwischen wichtigen und unwichtigen Nachrichten unterscheiden und erlaubte keine Priorisierung. Hinzu kam eine Verunsicherung beim Pflegepersonal, welches sich nicht sicher war, ob die Ärzte den automatisch versendeten Nachrichten Beachtung schenken. Da jedoch das Pflegepersonal verantwortlich ist, den Ärzten stets adäquate Informationen zur Verfügung zu stellen, wurden die Ärzte oftmals zusätzlich mündlich informiert. Hinzu kommen technische Probleme mit dem Pager-System selbst, welche ausschließlich unidirektional operieren, was es für Ärzte unmöglich macht, auf dem gleichen Weg auf Benachrichtigungen zu antworten.

Andere Autoren identifizieren Probleme bei der Einführung von neuen Systemen aufgrund zusätzlicher Arbeitslast durch hohen Einarbeitungsaufwand oder mangelnde Ressourcen, die insbesondere im Hinblick auf die spätere Administration und Wartung der laufenden Systems anfällt (Østbye et al. 2003; Hansen et al. 2006).

\section{Privatsphäre und Datenschutz}

Für elf der 67 identifizierten Systeme setzen sich die Autoren mit Bedenken bezüglich möglicher
Verletzungen der Privatsphäre und des Datenschutzes auseinander, beschränken sich dabei aber oftmals auf einen Hinweis auf Maßnahmen, welche die Einhaltung von Datenschutz und -sicherheit gewährleisten sollen. Derartige Maßnahmen umfassen beispielsweise die Anonymisierung der Benutzer- bzw. Patientendaten oder die verschlüsselte Datenübertragung (Anliker et al. 2004; Salvador et al. 2005; de Toledo et al. 2006; Ghinea et al. 2006). Fry und Lenert (2005) weisen in ihrer Studie jedoch darauf hin, dass der Grad der derzeit verfügbaren Verschlüsselung als zu gering anzusehen ist, um datenschutzrechtlich unbedenkliche Lokalisierung und Verfolgung von Patienten, Personal sowie Geräten zu garantieren. Salvador et al. (2005) versuchen Datenschutzprobleme durch den Einsatz eines komplexen Zugriffsrechtekonzeptes in den Griff zu bekommen, welches spezifische Zugriffsrechte für bestimmte Benutzergruppen verwaltet. In einer Studie über den Einsatz von tragbaren Kleincomputern in der Unfallambulanz soll Datenschutz beispielsweise dadurch sichergestellt werden, dass Patienteninformationen nicht permanent auf dem Gerät gespeichert sondern sofort nach deren Übertragung auf den Server gelöscht werden. Darüber hinaus ist das System in der Lage, das Sicherheitspersonal $\mathrm{zu}$ alarmieren, sobald gewisse Zonen innerhalb der Einrichtung verlassen werden (Gururajan 2004).

Andere Autoren versuchen durch Einbeziehen der Benutzer bestehende Bedenken hinsichtlich einer Verletzung der Privatsphäre bzw. des Datenschutzes abzubauen. Während die Überwachung der Arbeitsprozesse nach Østbye et al. (2003) grundsätzlich Ablehnung beim Klinikpersonal hervorruft, konnten Hansen et al. (2006) durch Einbeziehung der Nutzer in den Implementierungsprozess die Systemakzeptanz erhöhen. So wurde die Akzeptanz dadurch gesteigert, dass gewisse räumliche Bereiche (Pausenräume, Cafeteria etc.) von der Lokalisierung ausgeschlossen wurden. Durch derartige Maßnahmen können auch teils unbegründete Bedenken abgebaut werden. Beispielsweise berichten Sixsmith und Johnson (2004) von Erfahrungen mit Infrarot-Sensoren zur Erkennung von Stürzen in der Altenbetreuung. In diesem Fall gingen die Betroffenen fälschlicherweise davon aus, die Technologie wäre in der Lage, Überwachungsvideos zu konstruieren und Personen zu 
identifizieren, was folglich zu überzogenen Bedenken im Hinblick auf eine Verletzung der Privatsphäre führte.

Liszka et al. (2004) warnen hingegen vor ungelösten Datenschutzproblemen im Umgang mit Daten zur Lokalisierung insbesondere von Personen. Ihrer Ansicht nach existieren derzeit keinerlei Prinzipien für die angemessene Sammlung, Verwendung und Speicherung der Daten für den jeweiligen Anwendungsfall. Ferner weisen die Autoren explizit auf möglichen Datenmissbrauch hin, insbesondere durch die Möglichkeit der Verknüpfung mit Informationen aus anderen Datenbanken, und drängen auf eine Ausarbeitung von Richtlinien für den Umgang mit Problemen beim Datenschutz und der Gefährdung der Privatsphäre.

\section{Schlussfolgerungen}

Es kann festgehalten werden, dass sich Pervasive Computing noch in den „Kinderschuhen“ befindet, da nur ein geringer Teil der identifizierten Systeme bis dato klinische Test durchlaufen hat oder sich bereits im regulären Einsatz befindet. Als typische Einsatzfelder konnten erwartungsgemäß der häusliche und mobile sowie der innerklinische Bereich identifiziert werden. Der überwiegende Teil der Systeme verfolgt das Ziel, medizinische Verbesserungen im Bereich Prävention und Pflege zu realisieren. Bemerkenswert hoch ist auch der Anteil an Systemen, der sich organisatorische Verbesserungen zum Ziel setzen - ein weiterer Indikator für den steigenden Kostendruck im Gesundheitswesen.

Die qualitative Analyse hat gezeigt, dass sich überraschend wenige Autoren bereits während der Entwicklung mit möglichen Hindernissen oder negativen Faktoren für die spätere Systemeinführung auseinandersetzen. Dies ist insofern problematisch, da durch eine Berücksichtigung möglicher Einführungsbarrieren in frühen Phasen der Entwicklung die Chancen einer erfolgreichen Implementierung gesteigert werden könnten. Einige der analysierten Studien deuten dies - beispielsweise durch Einbeziehung der Anwender in den Entwicklungsprozess - an.

Diese Erkenntnisse verdeutlichen die Notwendigkeit interdisziplinärer Ansätze zur Einführung technologischer Innovationen im Ge- sundheitswesen, wie sie auch im Rahmen des PerCoMed-Projekts verfolgt werden. Eine erfolgreiche Kooperation von Systemherstellern und Anwendern unter Miteinbeziehung von Experten aus den Sozialwissenschaften reduziert die Gefahr einer rein technologiegetriebenen Systementwicklung. Stattdessen rückt, durch eine umfassende Abschätzung von Chancen und Risiken, der Anwender in den Mittelpunkt des Einführungsprozesses - eine, im gesellschaftlich hoch sensiblen Bereich der medizinischen Versorgung zentrale Voraussetzung für Projekte, die Technologien erfolgreich einführen wollen.

\section{Anmerkungen}

1) Gefördert vom Bundesministerium für Bildung und Forschung, Förderkennzeichen 16I1546.

2) Daneben werden in Orwat et al. (2008) die Systeme auch nach Nutzergruppen und Systemmerkmalen, d. h. Komponententypen, Typen der Datenerfassung, Gebrauch der Datenübermittlung und Systemfunktionen, kategorisiert sowie auch durch die Kombinationen verschiedener Kategorien ausgewertet.

\section{Literatur}

Anliker, U.; Ward, J.A.; Lukowicz, P. et al., 2004: AMON: a wearable multiparameter medical monitoring and alert system. In: IEEE Transactions on Information Technology in Biomedicine 8/4 (2004), S. $415-427$

Dadd, M.; Doyle, B.; Wilson, L. et al., 2002: Lessons learned from the Hospital Without Walls project. In: Journal of Telemedicine and Telecare 8/Suppl. 3 (2002), S. 11-14

Dahl, Y., 2006: „You have a message here“: Enhancing interpersonal communication in a hospital ward with location-based virtual notes. In: Methods of Information in Medicine 45/6 (2006), S. 602-609 de Toledo, P.; Jiménez, S.; del Pozo, F. et al., 2006: Telemedicine experience for chronic care in COPD. In: IEEE Transactions on Information Technology in Biomedicine 10/3 (2006), S. 567-573

Fry, E.A.; Lenert, L.A., 2005: MASCAL: RFID tracking of patients, staff and equipment to enhance hospital response to mass casualty events. In: AMIA Annual Symposium Proceedings (2005), S. 261-265

Ghinea, G.; Asgari, S.; Moradi, A. et al., 2006: A Jini-based solution for electronic prescriptions. In: IEEE Transactions on Information Technology in Biomedicine 10/4 (2006), S. 794-802 
Gururajan, R., 2004: A study of the use of handheld devices in an emergency department. In: Journal of Telemedicine and Telecare 10/Suppl. 1 (2004), S. 33-35

Hansen, T.R.; Bardram, J.E.; Soegaard, M., 2006: Moving Out of the Lab: Deploying Pervasive Technologies in a Hospital. In: IEEE Pervasive Computing 5/3 (2006), S. 24-31

Liszka, K.J.; Mackin, M.A.; Lichter, M.J. et al., 2004: Keeping a beat on the heart. In: IEEE Pervasive Computing 3/4 (2004), S. 42-49

Orwat, C.; Graefe, A.; Faulwasser, T., 2008: Towards Pervasive Computing in Health Care - A Literature Review. In: BMC Medical Informatics and Decision Making (eingereicht)

Østbye, T.; Lobach, D.F.; Cheesborough, D. et al., 2003: Evaluation of an infrared/radiofrequency equipment-tracking system in a tertiary care hospital. In: Journal of Medical Systems $27 / 4$ (2003), S. 367-380

Oyri, K.; Balasingham, I.; Hogetveit, J.O., 2006: Implementation of wireless technology in advanced clinical practice. In: Studies in Health Technology and Informatics 122 (2006), S. 730-733

Reddy, M.C.; Pratt, W.; McDonald, D.W. et al., 2003: Challenges to physicians' use of a wireless alert pager. In: AMIA Annual Symposium Proceedings (2003), S. 544-548

Salvador, C.H.; Carrasco, M.P.; de Mingo, M.A.G. et al., 2005: Airmed-cardio: a GSM and Internet services-based system for out-of-hospital follow-up of cardiac patients. In: IEEE Transactions on Information Technology in Biomedicine 9/1 (2005), S. 73-85

Sixsmith, A.; Johnson, N., 2004: A Smart Sensor to Detect the Falls of the Elderly. In: IEEE Pervasive Computing 3/2 (2004), S. 42-47

\section{Kontakt}

Andreas Gräfe

Institut für Technikfolgenabschätzung und Systemanalyse (ITAS)

Forschungszentrum Karlsruhe in der Helmholtz-

Gemeinschaft

Postfach 36 40, 76021 Karlsruhe

Tel.: +49 (0) 7247 / 82 - 4841

Fax: +49 (0) 7247 / 82 - 4806

E-Mail: andreas.graefe@itas.fzk.de

$《 》$

\section{Sektorenübergreifendes Informationsmanagement im Gesundheitswesen Neue Wege durch Pervasive Computing}

\author{
von Carsten Holtmann, Asarnusch Rashid, \\ Mercè Müller-Gorchs, Bernd Griewing, \\ Charlotte Kögerl und Volker Ziegler, For- \\ schungszentrum Informatik u. a.
}

\begin{abstract}
„Stroke Angel“" ist die erste der Fallstudien im Forschungsprojekt PerCoMed, bei der der Einsatz von Pervasive-Computing-Technologien zur Verbesserung des Informationsmanagements an der präklinisch-klinischen Schnittstelle in der Schlaganfallversorgung evaluiert wird. ${ }^{2}$ Im Rahmen dieses Teilprojektes erfassten Rettungsassistenten bei Verdacht auf Schlaganfall die Patientendaten leitfadengestützt mit mobilen Geräten am Notfallort und versandten diese bereits vor dem Transport des Patienten an das Zielkrankenhaus. Die Ärzte im Krankenhaus konnten sich durch diese Vorinformation auf den Notfall vorbereiten und eine reibungslose Übernahme des Patienten sicherstellen. Die PerCoMed-Forschungspartner flankierten zwei Jahre lang das Stroke-Angel-Projekt ${ }^{3}$ mit wissenschaftlichen Untersuchungen und führten sowohl technische als auch sozioökonomische und soziotechnische Analysen durch. In dieser Arbeit werden die Ergebnisse des Projektes vorgestellt und die Bedeutung von Pervasive Computing für ein sektorenübergreifendes Informationsmanagement diskutiert. ${ }^{4}$
\end{abstract}

\section{Informationsmanagement und Notfall- versorgung}

Der Schlaganfall ist ein zeitkritischer medizinischer Notfall. Noch deutlicher als beim akuten Herzinfarkt hängt beim akuten Hirninfarkt der Therapieerfolg stark von der Zeit des Therapiebeginns ab. Wird ein Schlaganfall rechtzeitig behandelt, kann der Gesundheitszustand des Patienten vollständig wiederhergestellt werden. Bei zu später Behandlung ist hingegen mit bleibenden Schäden, Behinderungen oder auch mit dem Versterben des Patienten zu rechnen (siehe Abb. 1).

Der Schlaganfall ist eine zerebrovaskuläre Erkrankung, die durch das Sterben der Nerven- 